\title{
SPATIALITÉS ET OBJETS VOTIFS
}

Le projet « Núcleo de Pesquisa dos Ex-votos »

José Cláudio Alves de Oliveira, Caroline Perrée

Éditions de l'EHESS | «Techniques \& Culture »

2018/2 n 70 | pages 126 à 139

ISSN 0248-6016

Article disponible en ligne à l'adresse :

https://www.cairn.info/revue-techniques-et-culture-2018-2-page-126.htm

Distribution électronique Cairn.info pour Éditions de l'EHESS.

(C) Éditions de l'EHESS. Tous droits réservés pour tous pays.

La reproduction ou représentation de cet article, notamment par photocopie, n'est autorisée que dans les limites des conditions générales d'utilisation du site ou, le cas échéant, des conditions générales de la licence souscrite par votre établissement. Toute autre reproduction ou représentation, en tout ou partie, sous quelque forme et de quelque manière que ce soit, est interdite sauf accord préalable et écrit de l'éditeur, en dehors des cas prévus par la législation en vigueur en France. Il est précisé que son stockage dans une base de données est également interdit. 


\section{Techniques \& Culture}

Revue semestrielle d'anthropologie des techniques

$70 \mid 2018$

Matérialiser les désirs

\section{Spatialités et objets votifs}

Le projet « Núcleo de Pesquisa dos Ex-votos »

José Cláudio Alves de Oliveira et Caroline Perrée

\section{(2) OpenEdition}

Journals

Édition électronique

URL : http://journals.openedition.org/tc/9746

DOI : $10.4000 /$ tc. 9746

ISSN : 1952-420X

Éditeur

Éditions de l'EHESS

\section{Édition imprimée}

Date de publication : 30 octobre 2018

Pagination : 126-139

ISBN : 2-7132-2751-6

ISSN : 0248-6016

Distribution électronique Cairn

\section{CAIRN INFO}

CHERCHER, REPÉRER, AVANCER

\section{Référence électronique}

José Cláudio Alves de Oliveira et Caroline Perrée, «Spatialités et objets votifs », Techniques \& Culture [En ligne], 70 | 2018, mis en ligne le 06 décembre 2020, consulté le 16 janvier 2019. URL : http:// journals.openedition.org/tc/9746; DOI : 10.4000/tc.9746 


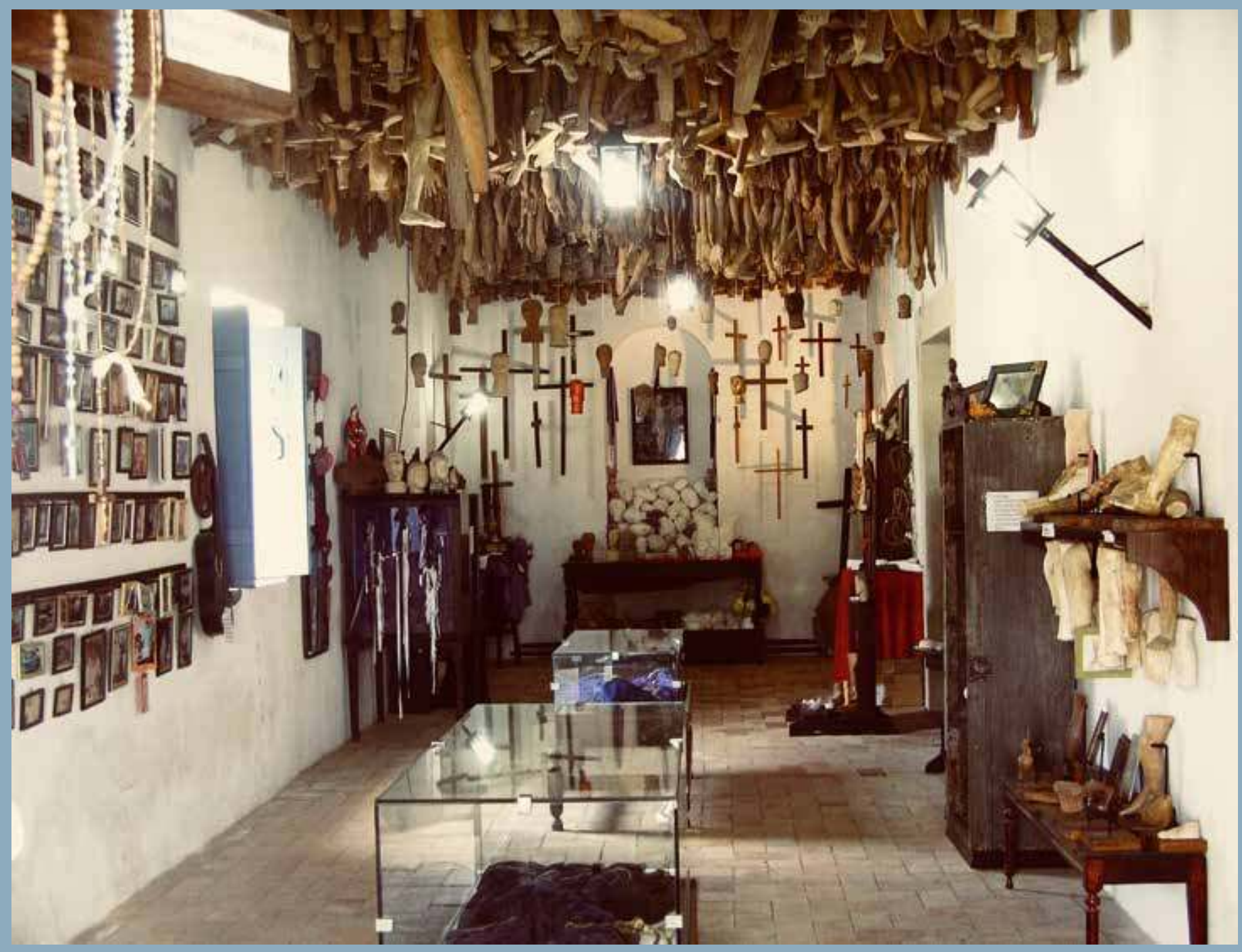




\section{Spatialités et objets votifs}

\section{Le projet « Núcleo de Pesquisa dos Ex-votos »}

Depuis 2005, l'Université fédérale de Bahia (UFBA) au Brésil consacre un projet d'études aux salles qui reçoivent les ex-voto. Appelées «Sala dos Milagres» (Salle des Miracles), elles sont situées dans la proximité d'un sanctuaire, à l'intérieur, à côté ou en dessous, et regroupent des objets hétéroclites aux origines diverses souvent extraits du quotidien. En 2008, le projet « Núcleo de Pesquisa dos Ex-votos» (NPE) coordonné par José Cláudio Alves de Oliveira, réunit 10 chercheurs et 38 étudiants. Partie du Brésil (2005-2011), la recherche s'étend aux pays d'Amérique centrale puis à l'Amérique du Nord (2011-2013) pour se concentrer sur le Mexique (de 2013 à nos jours). Le Brésil s'impose par le nombre de sanctuaires étudiés: 40 lieux saints qui s'échelonnent de l'État de São Paolo à celui de Alagoas en passant par les États de Minas Gerai, Goiás, Bahia et Sergipe. Ce sont au total $7802 \mathrm{~km}$ parcourus pour inventorier l'objet votif dans sa diversité, comprendre son usage dévotionnel, et rendre compte des circonstances de son dépôt et de son exposition.

Cette enquête à l'échelle d'un continent donne lieu à 5 publications collectives pluridisciplinaires (Oliveira \& Prêtre 2018; Oliveira 2016, 2017; Oliveira, Luke Agraz \& Perrée 2017; Oliveira, Magno \& Martin 2014), et à des sites Internet retraçant les avancées des recherches et constituant une base de données iconographique ${ }^{1}$. Au fur et à mesure, les perspectives d'analyse évoluent d'un inventaire classificatoire des formes et contenus votifs à leur étude muséographique. Observé in situ, l'ex-voto est analysé comme un objet mémoriel, informatif et communicationnel. Comment les spatialités dans lesquelles il est intégré rendent-elles compte de ces dimensions, et que nous apprennent-elles sur l'objet votif? 
1. Carte des sanctuaires étudiés au Brésil

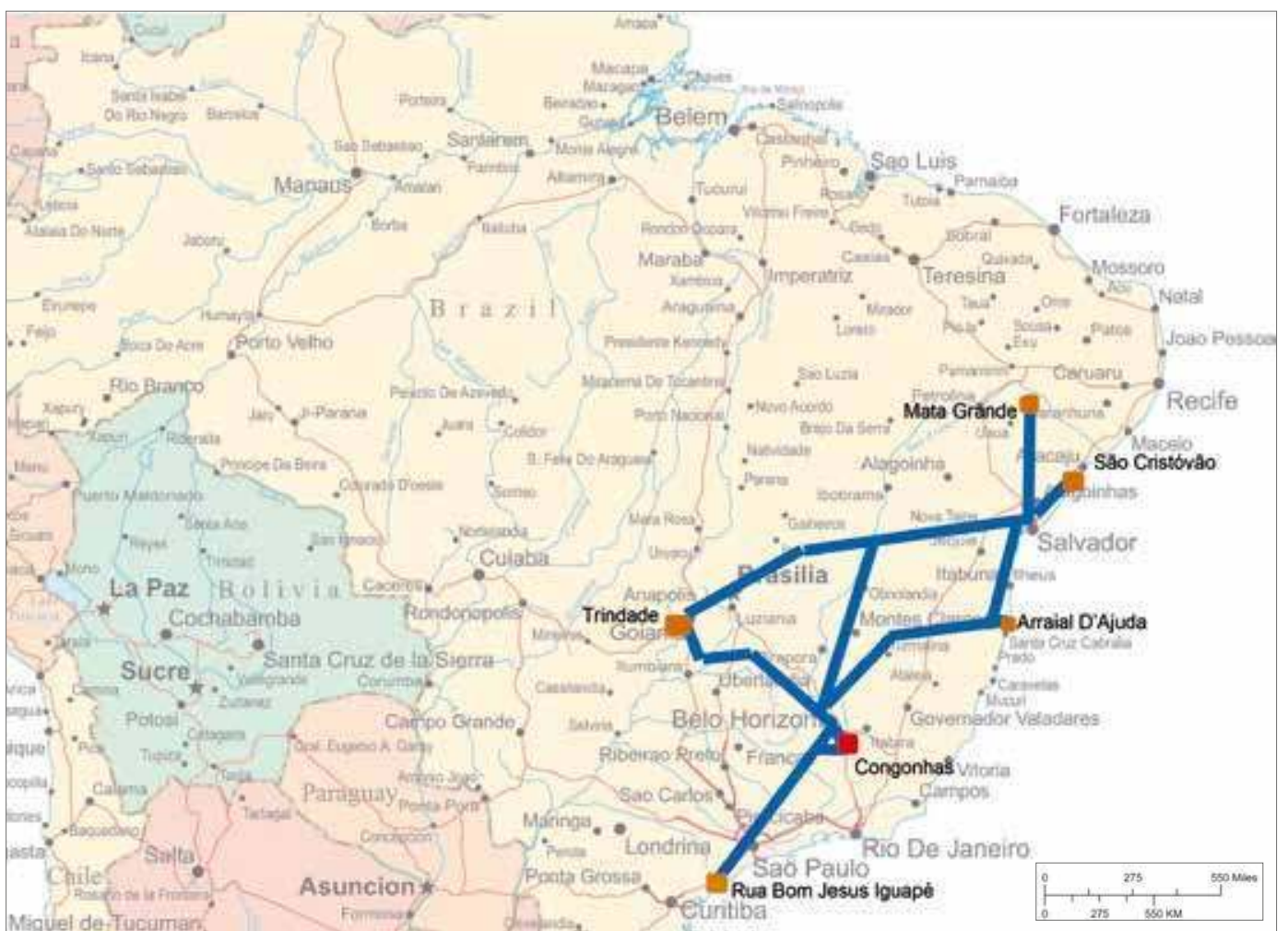

\section{Ies Salles des Miracles}

Au-dessus des visiteurs sont suspendues des dizaines de membres anatomiques en bois, au fond de la salle s'accumulent des têtes en cire, alternant avec des croix déposées après le pèlerinage. À gauche, une paroi de cadres contient des images d'intercesseurs divins. En face, des étagères sur lesquelles sont disposés des ex-voto anatomiques en bois et en cire. Au milieu, deux boîtes contenant plusieurs types d'ex-voto, dont des sculptures (image d'ouverture). Au Convento do Carmo à São Cristóvão, la disposition semble reposer sur des typologies votives préétablies. Pourtant, celles-ci sont l'œuvre de pèlerins qui, comme à Mata Grande, à Matosinhos ou à Chalma et à San Juan de los Lagos au Mexique, sont libres d'accrocher leurs dons où bon leur semble. Et ils les suspendent selon des catégories formelles. L'ex-voto se donne alors à voir sur le mode de la répétition du même au même. Son accumulation forme une immense prière gratulatoire ou propitiatoire matérialisée, qui exalte sa force communicationnelle avec le divin et avec les hommes. En effet, il manifeste à la fois la preuve d'un miracle reçu et le témoin d'une promesse accomplie aux yeux de tous (Magno dos Santos 2016). 


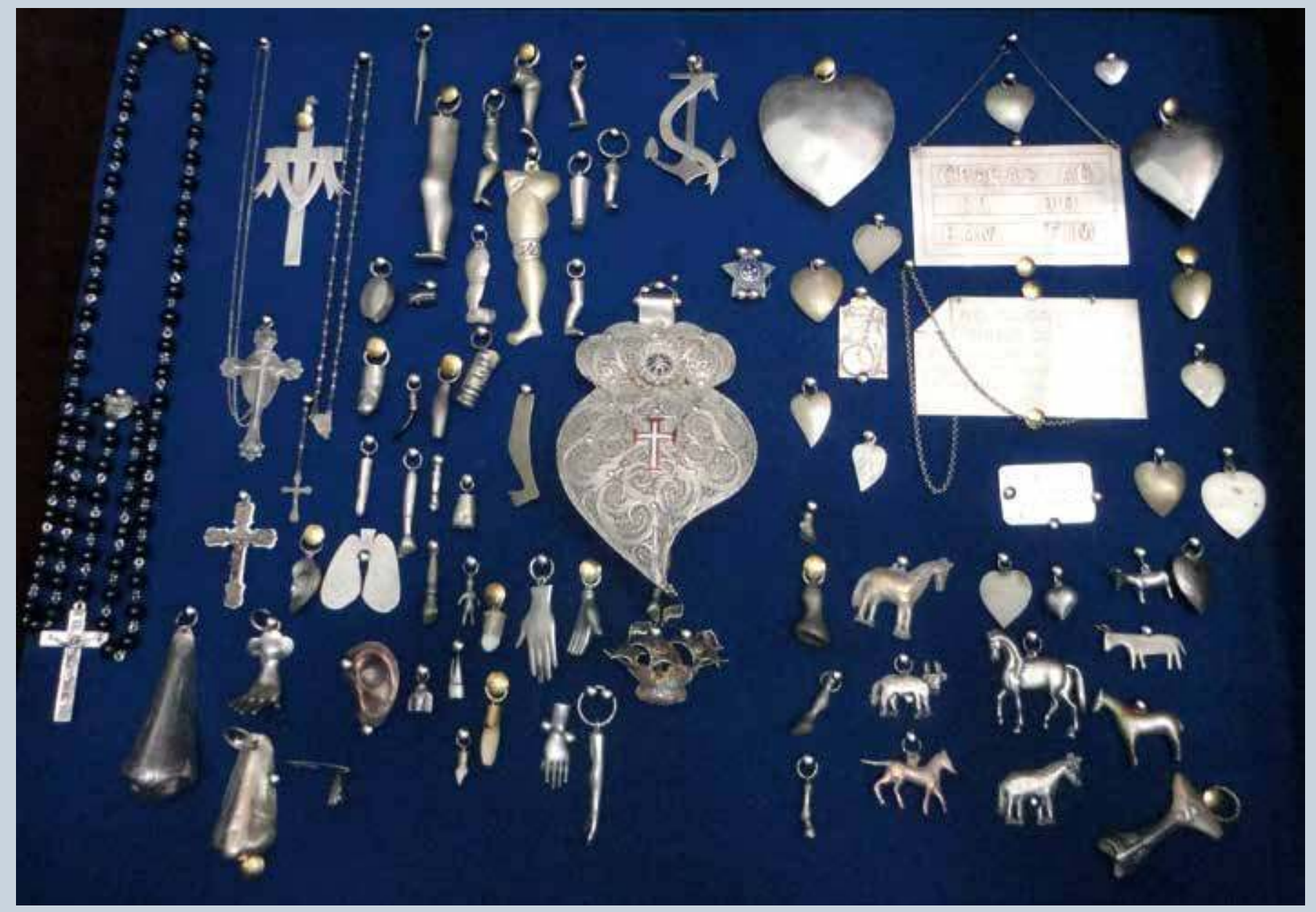

2. Vitrine votive du musée du Senhor de Bomfim à Salvador de Bahía, Brésil 

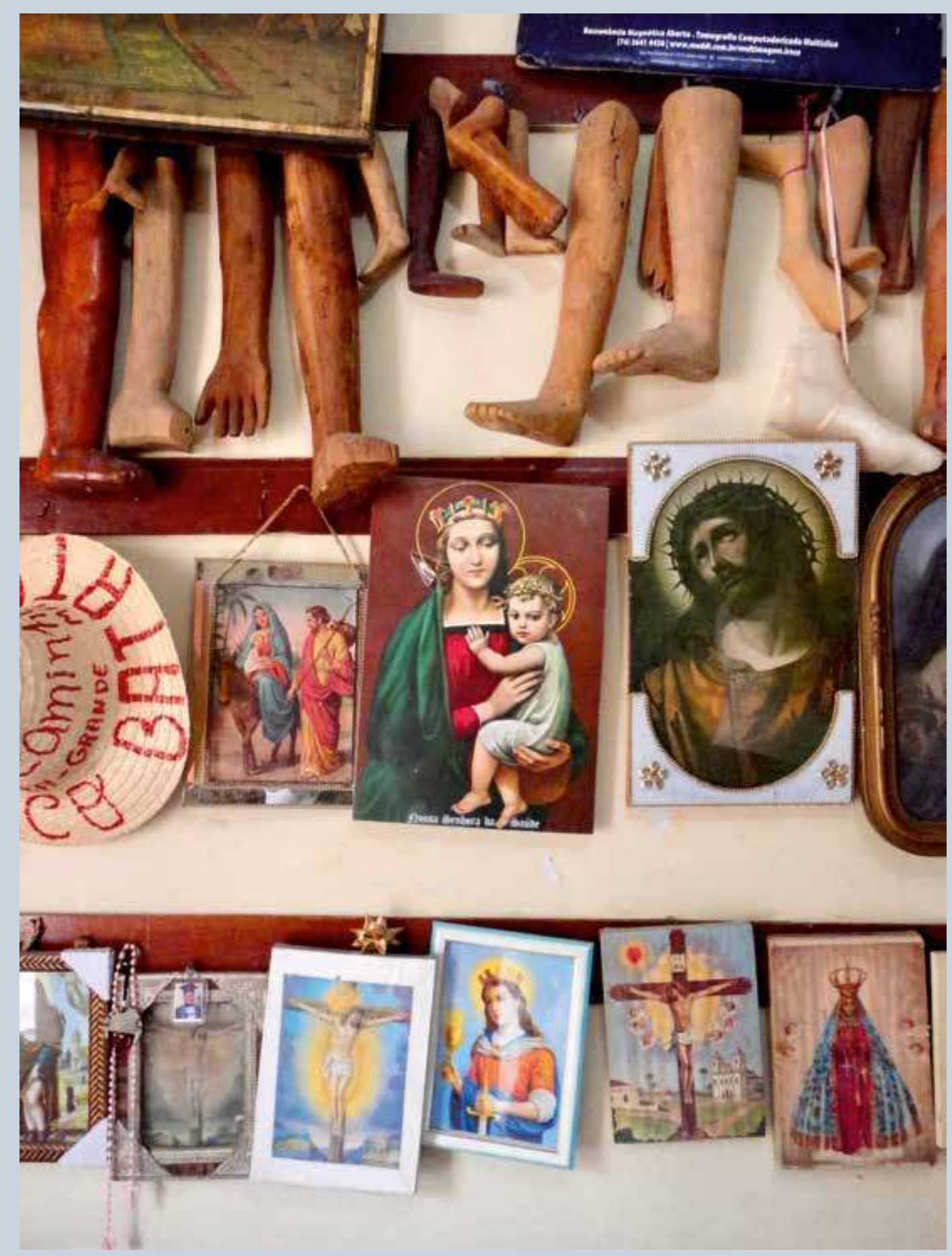
Ailleurs, comme à Trindade ou à Nossa Senhora Aparecida à São Paolo, c'est le clergé qui reçoit et sélectionne les ex-voto pour les exposer ou pas, selon une muséographie fondée sur des typologies une nouvelle fois formelles. Ces dispositions par séries visuelles apparentent la Salle des Miracles à un musée, comme celui du Senhor de Bomfim à Salvador de Bahía, à la différence près que la salle ne comporte pas de base de données. Celle du musée est établie par un muséologue, que le clergé invite pour organiser l'exposition. Genivalda Cândido, du NPE, a été engagée par le sanctuaire de Nosso Senhor do Bomfim. Pour ces trois acteurs de la spatialisation votive, croyant, clergé et muséographe, l'objet exposé est le vecteur matériel et communicationnel de la relation invisible nouée entre l'humain et le divin. Son accrochage repose sur une saturation visuelle des miracles en présence.

\section{Le lieu d'une mémoire sociale}

Dans la salle de la petite église de Nossa Senhora dos Milagres de Brotas, à Milagres dans l'État de Bahia, sont alignés des jambes, des bras et des pieds en bois et en cire. Au-dessous, des représentations de figures divines encadrées et ordonnées selon leurs dimensions. Cette disposition met en scène l'univers duel des acteurs de la démarche votive, et illustre sa matérialité. D’un côté, le monde divin avec sa kyrielle d'intercesseurs, Christ, Vierge ou saint selon la dévotion de chacun. De l'autre, l'humain, dont l'invocation est symbolisée par un objet qui se substitue à lui. Les objets en série ressortent d'une pratique partagée par tous, comme en atteste l'usage de membres anatomiques extrêmement répandus sur le continent, nommés "grâces » ou «miracles» par les croyants. Ils entrent dans la typologie des ex-voto anthropomorphes (Oliveira 2016), manufacturés (Boullet 1978) ou figuratifs (Sigal 1985); la diversité des nominations soulignant la difficulté à catégoriser l'objet votif. Mais leur identification est immédiate par les croyants, car, même si les ex-voto sont souvent décrits comme hétéroclites, certains sont identifiés d'emblée comme votifs, parce que leur usage est traditionnel. Les membres anatomiques sont sans doute les plus anciens ex-voto répertoriés comme tels. Ils se distinguent par la diversité de leur matériau de confection, qui fixe une échelle de valeur dans la grâce à payer: en terre cuite dans les cultures antiques grecque et étrusque (Regnault 1952), mais aussi en marbre pour les plus chers. Du Moyen Âge à nos jours, en Europe comme sur le continent américain, les plus onéreux sont en or ou en argent; les plus courants en cire, en bois et en métal. D'autres objets en série ressortent, eux, d'un usage régional, à l'instar des images à l'effigie de la Vierge, du Christ et des saints présentes également dans les maisons et oratoires du NordEst du Brésil. Ils font partie d'une mémoire sociale, que met au jour la spatialité de la Salle des Miracles. Témoins d'une «mémoire collective» (Halbwachs 1994) de la foi, transmise par la pratique de génération en génération, ils forment autant de témoignages sur les pèlerins, leur quotidien et leurs croyances.
Salle des Miracles de Nossa Senhora dos Milagres de Brotas, à Milagres, état de Bahia, Brési 
Photographie votive. Salle des Miracles de Bom Jesus do Matosinhos, à Congonhas, état de Minas Gerais, Brésil.
Certains objets revêtent un caractère plus subjectif, parce qu'ils personnalisent la mémoire individuelle ainsi présentée. C'est le cas de la photographie, qui est l'un des dons les plus communs aujourd'hui en Amérique latine pour son caractère représentatif, son faible coût et sa réalisation facile. L'efficacité qui lui est attribuée repose sur la croyance dans le pouvoir guérisseur d'un simulacre à l'identique: parce qu'il est figuré grâce à son image, le récipiendaire peut faire l'objet d'une intervention miraculeuse. La photo de la petite fille déposée dans la salle de Bom Jesus do Matosinhos, à Congonhas ne comporte pas de date. Elle est anonyme mais elle atteste d'un «ça a été » et d'un certificat de présence (Barthes 1980), renforcée par la tresse qui établit un lien vivant avec l'enfant, et qui affirme la présence individuelle dans le collectif des demandes et remerciements réunis dans la Salle des Miracles. La chevelure appartient également à cette mémoire sociale de la dévotion car sur le plan sémiotique, la tresse rappelle les cheveux coupés en paiement de la promesse faite, pratique identifiable par tous les croyants. Ce type de dispositif votif associant don figuratif et don métonymique est très commun au Brésil comme au Mexique. Le vêtement peut aussi prendre la place de la tresse comme métonymie du corps du donateur.

\section{Objet figuratif, objet métonymique, la mémoire du corps}

Dans la Salle des Miracles de Santa Terezinha, à Mata Grande, dans l'état d'Alagoas, des tresses de cheveux sont accrochées à des mains en bois sculpté. Les unes et les autres sont offertes pour symboliser le corps malade ou guéri. À travers ce rite de substitution, l'ex-voto peut prendre une forme figurative ou métonymique qui explique la croyance dans son efficacité. L'ex-voto anatomique engage les deux: figuratif, il ressemble au membre corporel, c'est pourquoi il peut faire l'objet d'une action. Sa fabrication et sa manipulation impliquent le contact du corps du donateur avec son simulacre, qui est mis par la suite dans la proximité du corps guérisseur de l'image divine, à l'intérieur de la Salle des Miracles. La matière modelable du bois et de la cire augure de la transformation du mal en guérison, de la chair meurtrie qui prend la forme d'un désir (Didi-Huberman 2006).

La chevelure se présente, elle, comme une «métonymie matérielle» (Dehouve 2013). Puisque «la personnalité d'un être est indivise et réside tout entière dans chacune de ses parties » (Hubert, Mauss 1999), à travers l'opération de transfert matériel activée par le mécanisme d'association contiguë (Hémond 2013), la chevelure offerte établit une continuité d'action entre le corps et ses parties. En Amérique latine, ce don très courant est lié à une manda ou promesa, à savoir une promesse à accomplir si le divin est intervenu en faveur du croyant, conformément à la démarche votive. La plupart du temps, le clergé se défait rapidement de ces dons soit par la fabrication de perruques pour les Vierges du sanctuaire ou pour des personnes atteintes d'un cancer, soit par leur vente à des salons d'esthétique. Objet biographique, l'ex-voto conserve la mémoire du corps, celui du donateur à soigner, celui du saint guérisseur. 


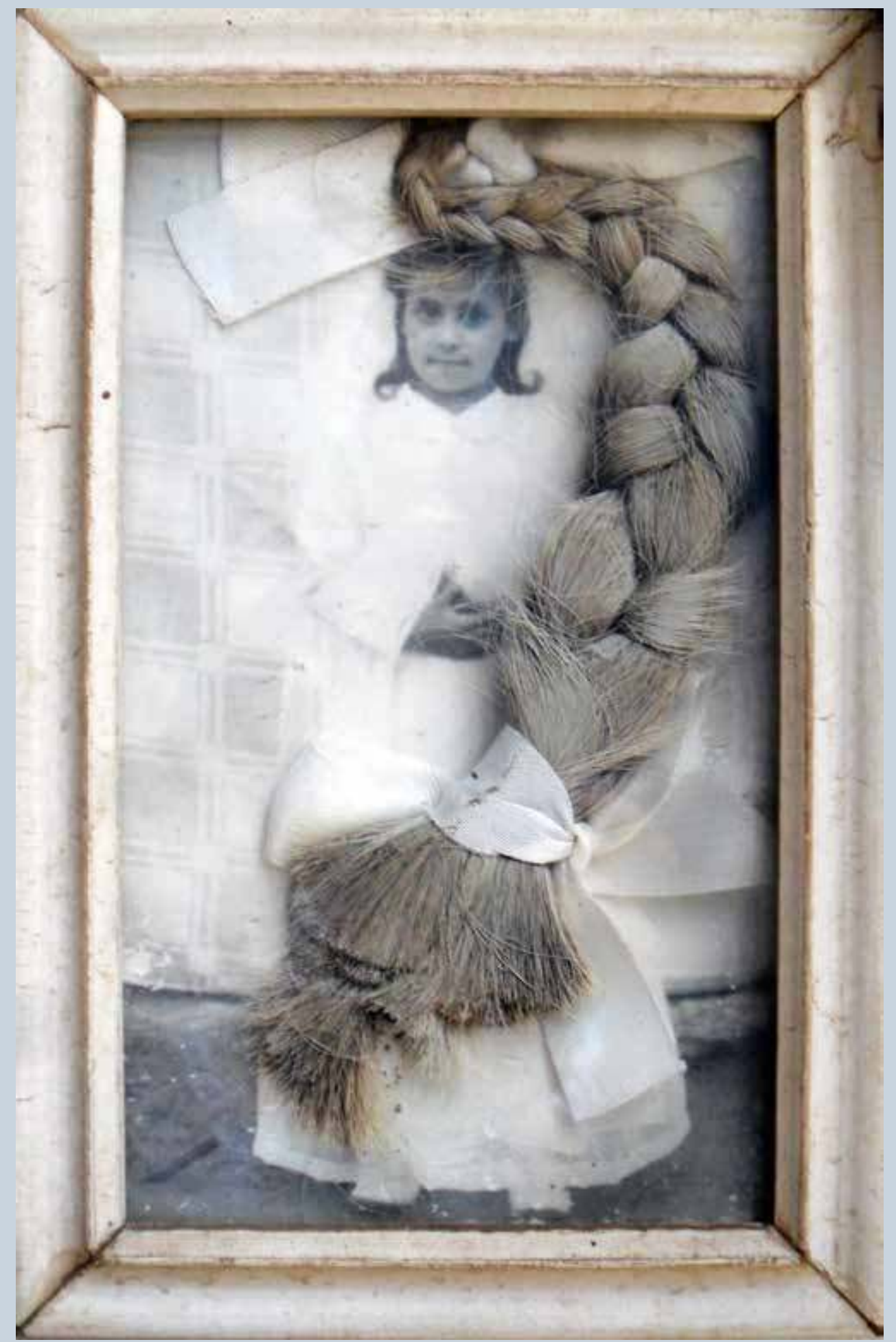




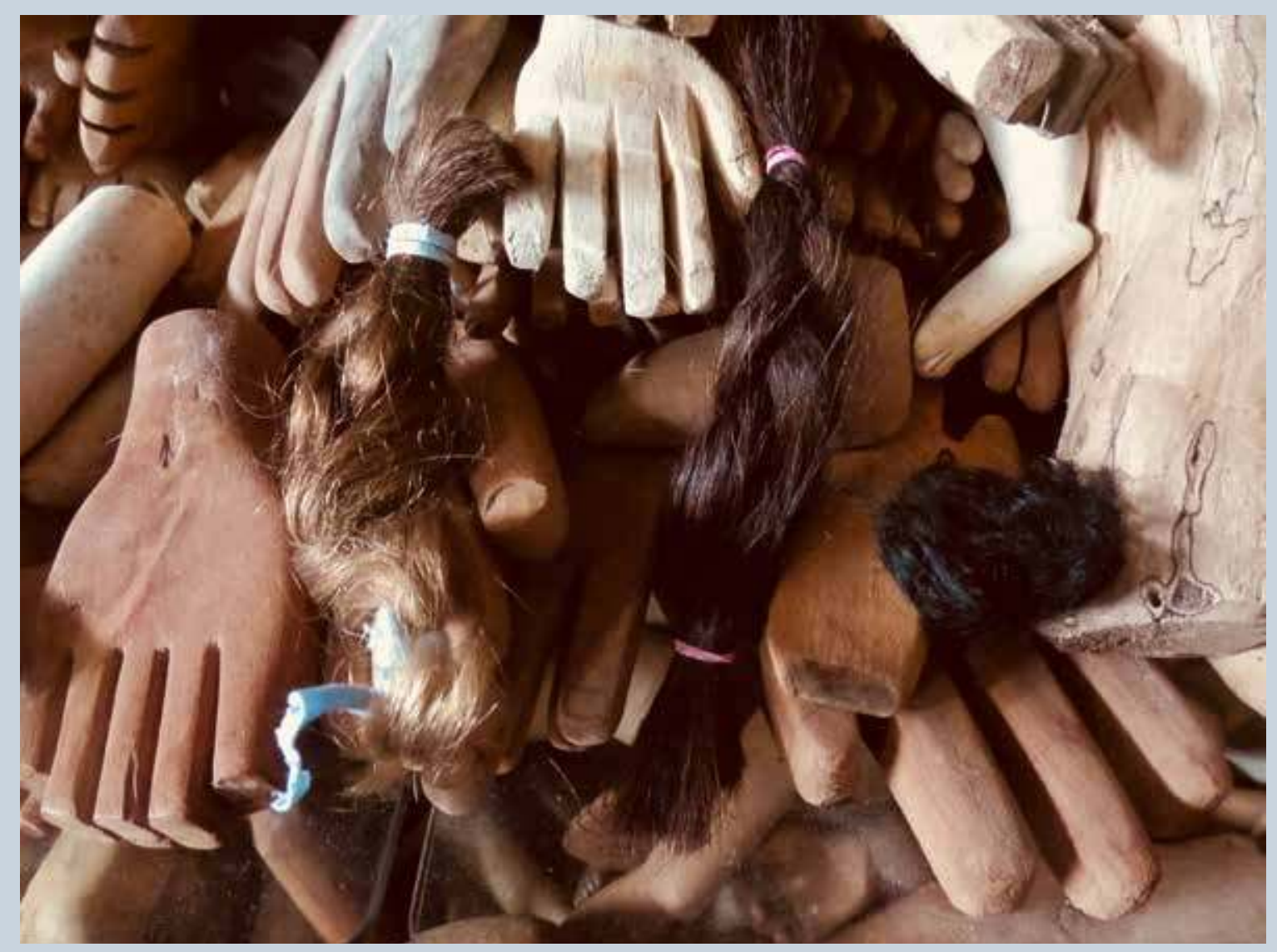

5. Membres anatomiques et mèches de cheveux votifs. Salle des Miracles du sanctuaire de Santa Terezinha, à Mata Grande, État d'Alagoas, Brésil 


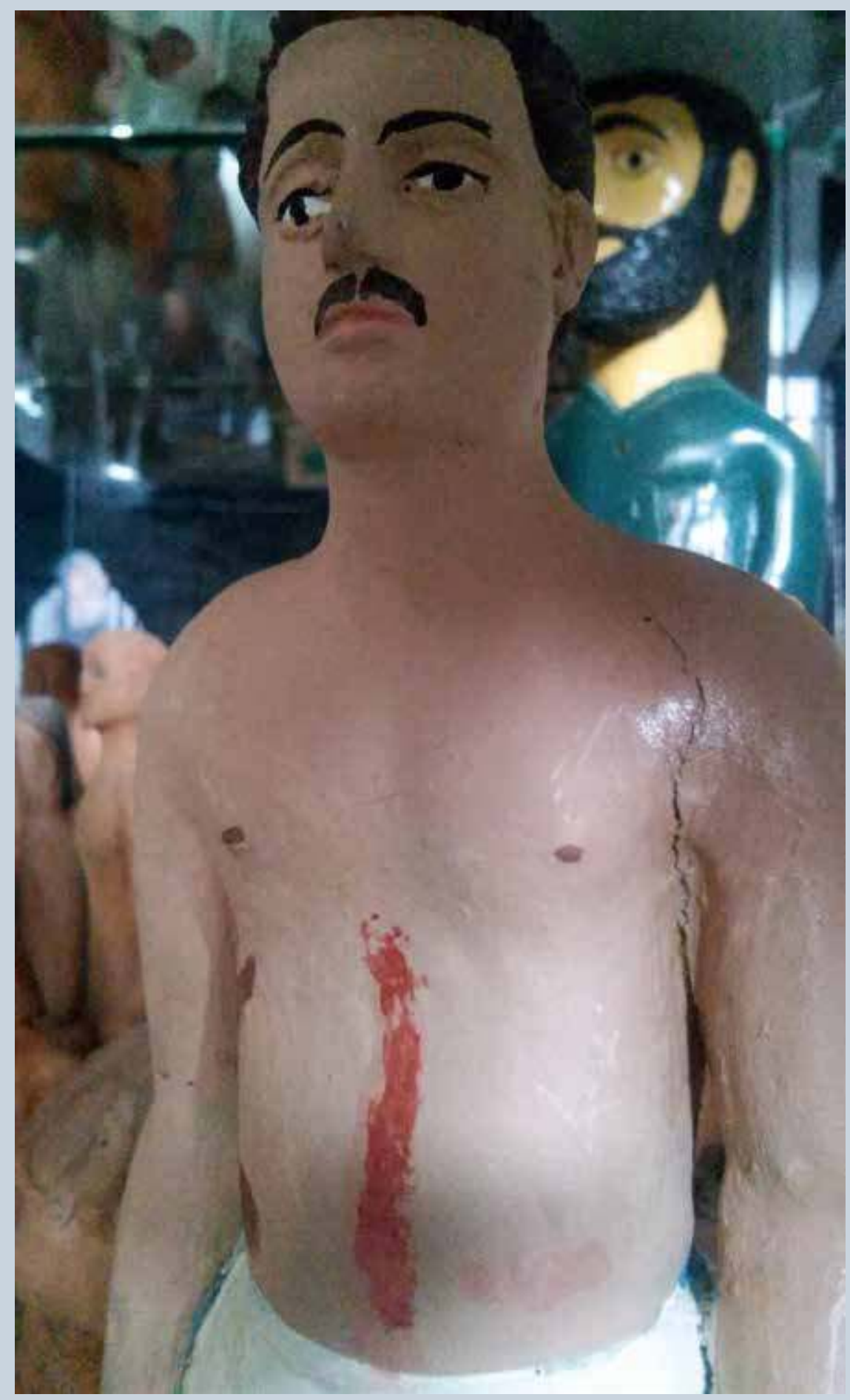

6. Buste d'homme dans la Salle des Miracles de Santa Terezinha, à Mata Grande, État de Alagoas, Brésil 


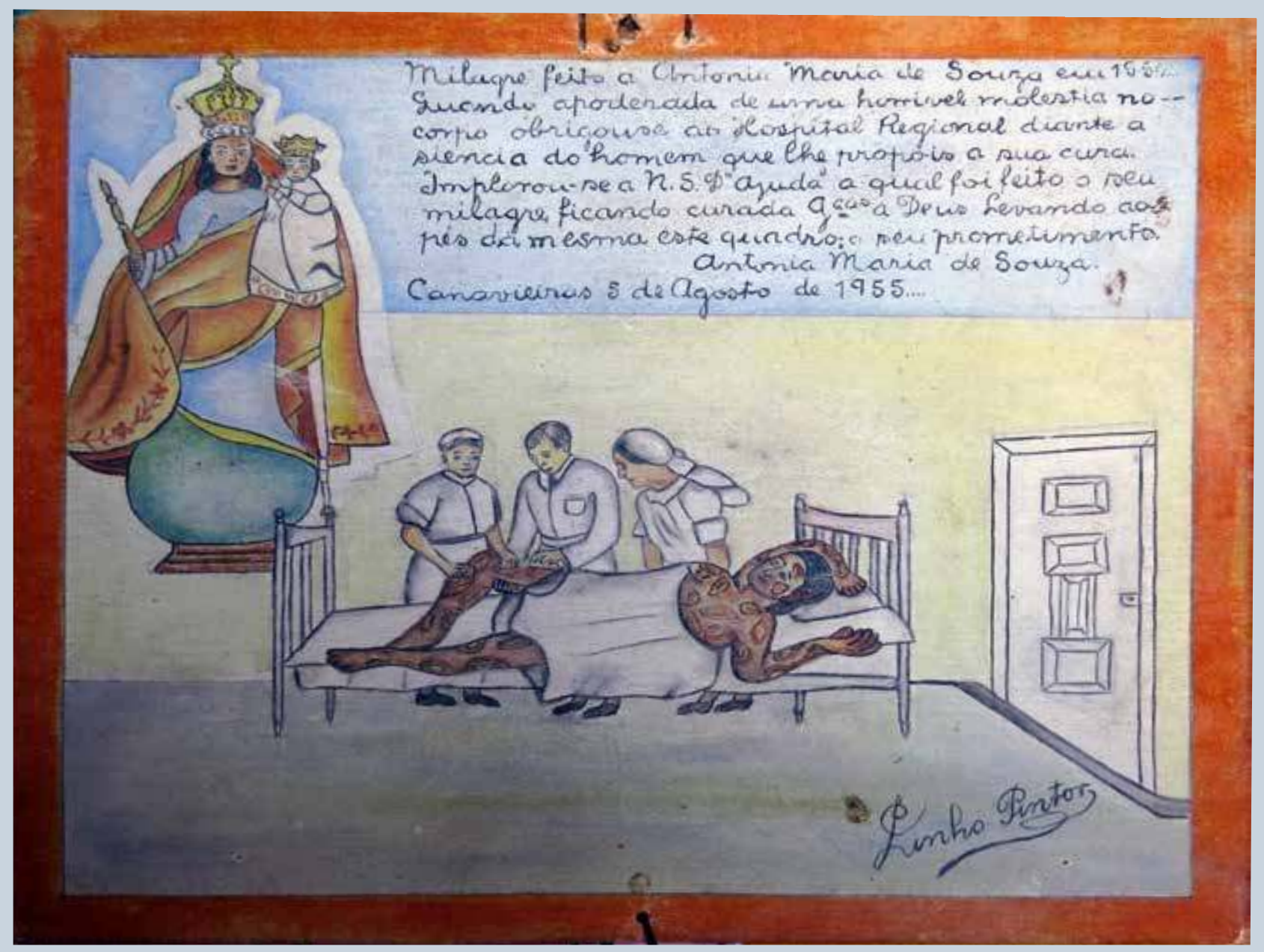

7. Ex-voto peint de la Salle des Miracles de Nossa Senhora D'Ajuda, à Arraial d'Ajuda, État de Bahia, Brésil 


\section{Objet sémiotique d'une histoire collective}

Une statue polychrome d'homme en position hiératique, réalisée en gypse polychrome trône dans la Salle des Miracles de Santa Terezinha, à Mata Grande. Le teint clair, la peau rose, la pilosité brune, le pantalon blanc, ces couleurs contrastent avec la ligne rouge qui balafre son abdomen. Le rouge sur le corps nu revêt une valeur indicielle (Peirce 1978) de la souffrance propre à communiquer avec le saint pour lui indiquer le lieu de la blessure à soigner. Dans un système de double énonciation, créée par l'exposition de la statue, le rouge communique aussi aux visiteurs le degré de la souffrance et le danger encouru symbolisés par la longueur de la ligne. Le trait ainsi tracé personnifie une statue peut-être fabriquée en série, individualise la douleur, conserve la mémoire de l'événement souffert par celui qui l'a déposée, lui conférant une dimension éminemment sociale et collective. Par sa puissance de communication iconographique (Vovelle 1979) et hors de toute considération esthétique, l'objet engage une analyse sémiotique de la souffrance, qui révèle une mémoire sociale du corps votif. Tandis que son exposition dans la salle mêle les histoires individuelles pour constituer une histoire collective.

\section{Objet de " folkcommunication "}

Dans la Salle des Miracles de l'église de Nossa Senhora D'Ajuda, à Arraial d'Ajuda dans l'État de Bahia, la Vierge reçoit une peinture dont le caractère miraculeux est illustré par le corps d'une femme couvert de plaies:

Miracle fait à Antonia Maria de Souza en 1950... alors qu'elle souffrait d'une horrible gêne sur son corps, elle a été prise en charge par l'hôpital régional grâce à la présence de l'homme qui l'a aidée à guérir. Elle a imploré N. S. D'Ayuda de l'aider. Celle-ci a fait le miracle en la guérissant grâce à Dieu. Elle dépose sa promesse aux pieds de l'image. Antonia Maria de Souza. Canavieiras le 5 août 1955... Signé par le peintre Linho.

Le lien étroit entre folklore et communication populaire a donné le jour au concept de «folkcommunication», défini par Beltrão comme «un processus d'échange d'informations et d'expression d'opinions, d'idées et d'attitudes des masses par le biais d'agents et de moyens directement ou indirectement liés au folklore » (Beltrão 1971) ${ }^{2}$.L'ex-voto, conçu comme un objet de communication, ressort de la folkcommunication sur les plans textuel et iconographique. En effet, sur l'image, les proportions ne sont pas respectées à escient pour mettre en valeur le corps de la femme, qui est l'objet du miracle. Quant au texte, il est ponctué de fautes d'orthographe: les mots « horrible», «présence », l'abréviation du nom du saint et le terme "promesse » employé à la place d'« engagement ». Pour autant, le message est compréhensible et illustre la spontanéité 
de la communication directe entre l'humain et le divin. L'ex-voto, plus que tout autre média classique, journaux, télévisions, radios et musées, permet au croyant de s'exprimer avec une liberté totale (Oliveira 2015). La spatialité de la Salle des Miracles orchestre une mise en scène de l'ex-voto, qui donne à voir et à lire les réalités cachées des croyants, en leur ouvrant un espace de parole qu'ils n'auraient pas ailleurs.

\section{Notes}

1. Sites à consulter: https://www.nucleodepesquisadosex-votos.org/; https://projetoex-votosdobrasil.net/

2. Le texte de l'ex-voto et la citation de Luis Beltrão en portugais sont traduits par Caroline Perrée.

\section{Les auteurs}

Caroline Perrée est docteure et chercheuse en histoire de l'art au CEMCA à Mexico. Après un doctorat portant sur l'ex-voto dans l'art contemporain, ses recherches actuelles s'inscrivent dans le champ de l'anthropologie de l'image par l'analyse des objets votifs et des images dévotionnelles, et leurs interactions avec les créations artistiques contemporaines, notamment au Mexique. Elle travaille plus généralement sur les questions de temporalité en art à travers l'analyse des continuités et des subversions dans la création des images.

José Cláudio Alves de Oliveira est chercheur en communication et culture contemporaine à l'Université fédérale de Bahia (UFBA) au Brésil. Il a réalisé son doctorat en communication et technologies à l'Université de Minho au Portugal. Il est actuellement professeur associé III du département de muséologie de l'UFBA et coordonne le « Núcleo de Pesquisa dos Ex-votos» et le «Projeto Ex-votos du Mexique». Il est professeur titulaire des programmes de master en science des informations et en muséologie à l'UFBA.

\section{Iconographie}

Image d'ouverture. Salle des Miracles du Convento do Carmo, à São Cristóvão, état de Sergipe, Brésil. @ Photo de J.C.A. de Oliveira.

1. (C) cartograf.fr.

2 à 6. (C) Photos de J.C.A. de Oliveira.

7. (C) Photographie de Flávia Maciel.

\section{Références}

Barthes, R. 1980 La Chambre claire. Note sur la photographie. Paris: Gallimard

Beltrão, L. 2004 [1971] Folkcomunicação: Teoria e Metodologia. São Bernardo do Campo: UMESP.

Boullet, F. \& C. 1978 Ex-voto marins. Genève: Éditions Maritimes et d'Outre-Mer.

Dehouve, D. 2013 «Les métaphores comestibles dans les rituels mexicains », Amérique Latine Histoire et Mémoire. Les Cahiers ALHIM 25. [En ligne] : http://journals. openedition.org/alhim/4675.

Didi-Huberman, G. 2006 Ex-voto image, organe, temps. Paris: Bayard

Halbwachs, M. 1994 [1925] Les Cadres sociaux de la mémoire. Paris: Albin Michel.

Hémond, A. 2013 « Métaphores temporelles et offrandes alimentaires: pratiques d'élaboration du sens rituel», Amérique Latine Histoire et Mémoire. Les Cahiers ALHIM 25. [En ligne] : http://journals.openedition. org/alhim/4547. 
Hubert, H. \& M. Mauss 1999 [1902-1903] «Esquisse d'une théorie générale de la magie», Sociologie et anthropologie. Paris: PUF («Quadrige»).

Magno dos Santos, G. 2016 «Uma abordagem da estética religiosa: Ex-votos bibliográficas da Básilica do Bomfim mémoria social» in J.C.A. de Oliveira dir. Ex-votos do Brasil: Arte e folkcomunicação. 1. Salvador: Quarteto.

Oliveira, J.C.A. de dir. 2015 Ex-votos das Américas Comunicação e memória social. 1. Salvador: Quarteto.

Oliveira, J.C.A. de dir. 2016 Ex-votos do Brasil : Arte e folkcomunicação. 1. Salvador: Quarteto.

Oliveira, J.C.A. de dir. 2017 Ex-votos das Américas: Comunicação e memória social. 2. Curitiba: CRV.

Oliveira, J.C.A. de \& C. Prêtre dir. 2018 Se eu quiser falar com Deus: História e lugares dos ex-votos. 1. Curitiba: CRV.
Oliveira, J.C.A. de, Luque Agraz, E. \& C. Perrée 2017 Ex-votos do México: tradição e transgressão. 1. Curitiba: CRV.

Oliveira, J. C. A. de, Magno, G. \& M.L. Martins dir. 2014 Memória e Epigrafia. 1. ed. Salvador: Vento Leste.

Peirce, Ch. S. 1978 Écrits sur le signe (rassemblés, traduits et commentés par G. Deledalle). Paris: Seuil.

Regnault, F. 1952 «Les Ex-voto polysplanchniques de l'antiquité », Bulletin de la société française de la médecine. Paris: 135-150

Sigal, P. A. 1985 L'Homme et le miracle dans la France médiévale (XI'-XII ${ }^{e}$ siècles). Paris: Éditions du Cerf.

Vovelle, M. 1979 Iconographie et histoire des mentalités. Paris: CNRS.

\section{Pour citer l'article}

Oliveira, J.C.A. de \& C. Perrée 2018 «Spatialités et objets votifs. Le projet "Núcleo de Pesquisa dos Ex-votos" », Techniques\&Culture 70 «Matérialiser les désirs. Techniques votives», p. 126-139. 